\title{
A Jump Diffusion Analysis for American and European Equity Markets in the beginning of the Corona Pandemic
}

\author{
Matthias Bernhardt
}

Munich, Germany.

\begin{abstract}
This paper deals with the analysis of jumps in equity markets in conjunction with the Corona Pandemic in the beginning of 2020. The aim is to identify, analyse and compare jumps in both American and European stock markets using SX5E Index and SPX Index, with jumps measured by Kou's Double Exponential Jump Diffusion Model. To the best of our knowledge this is the first paper which applies a pure time series model to analyse stock market behavior in terms of jumps using intraday data.

The result is that jumps in both markets have similiarities and differences in terms of model behavior before, during and after the V-shaped market movement early 2020.
\end{abstract}

Keywords: COVID-19, time series analysis, stock market jumps, Double Exponential Jump Diffusion Model.

\section{INTRODUCTION}

Since the outbreak of the Corona Pandemic in the beginning of 2020 the world is upside down. Curfews, lockdowns and quarantine regulation determine everyday life in most countries worldwide, which has a significant impact on the global economy.

*Address correspondence to this author at Munich, Germany;

E-mail: bernhardt_matthias@gmx.de
According to the Global Economic Prospects (World Bank, 2021) the Corona pandemic caused a recession whose depth was exceeded only by World War One and World War Two as well as the Great Depression. Of course the outbreak or the pandemic, respectively, can also be observed in equity markets.

Fig. (1) shows both the S\&P Index and the Eurostoxx 50 Index on a daily basis starting in the beginning 2020 until June 2020 . At the outbreak we observe a sharp downward move, followed

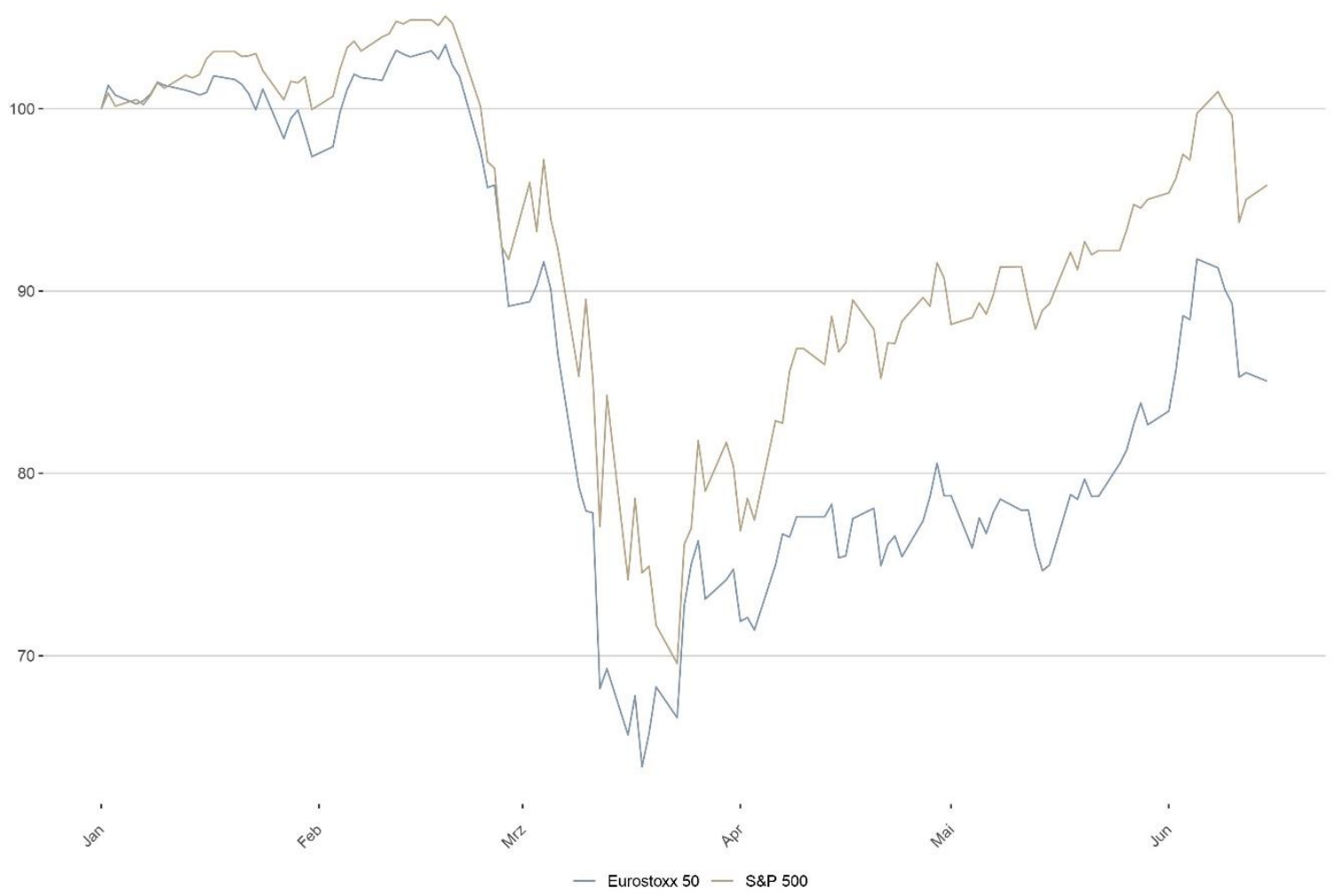

Fig. (1). Eurostoxx 50 and S\&P 500 Index from January to June 2020. 
by a steady recovery in both markets. Following (Sheiner \& Yilla, 2020) we can observe a more or less W-shaped recovery for the Eurostoxx 50 and a Nike Swoosh-Shaped recovery for the S\&P Index. However, in addition to this visual difference, we want to investigate the behavior of the two markets in more detail in terms of a jump diffusion analysis. To the best of our knowledge this is the first study which deals with a pure time series analysis of the Corona pandemic comparing US and European equity markets.

The rest of this work is organzied as follows. The next section gives a literature review regarding the impact of the pandemic on financial markets. Section 3 describes the methodology and data used in this paper. In section 4 we present and discuss the results of our analysis. Section 5 concludes and gives implications for policy makers and asset manager.

\section{LITERATURE REVIEW}

Although the Corona pandemic is not over yet there is starting a literature from different economical perspectives. In sum, the majority of studies try to link uncertainty to the COVID19 crisis by different approaches.

(Czeczeli, et al., 2020) take a macroeconomic view on the socio economic situation of 25 European countries before the outbreak of the virus and ask if these economic situations can be related to different economic behavior during the crisis.

The initial assumption is that there is a correlation between economic short-term behavior (measured as labour mobility, unemployment rate, industrial production and government bond spreads) during the first four months of the crisis and the state of public finances, income distribution and external vulnerabilities in each country prior to the outbreak of the crisis. They answer the question by forming a hierarical cluster analysis based on the latter variables and find seven clusters.

The authors come to the conclusion that the corona crisis as a exogenous shock has not the same effect as a endogenous shock. However, there is no clear seperation in terms of behavior of the short term indicators when comparing different clusters, but all clusters have in common that the decrease in labour mobility shows a high correlation with the reduction of industrial production, not with unemployment. Moreover, for most countries the indicators show a correction by June 2020 to their initial growth path. When looking at the stock market we can see that the European stock market did not adapt this observed correction. Therefore, we can carefully conclude that capital market participants did not at least fully adapt these findings.

Zooming closer to the effects of the pandemic to capital markets, (ElFayoumi \& Hengge, 2021) apply a similar approach as (Czeczeli, et al., 2020) by comparing pre crisis macro levels with portfolio flows during the crisis. One of the key findings of their study is that both the pandemic itself and government responses to the shock impact the supply curve as well as the demand curve of capital markets. So, they observe empirically what (Czeczeli, et al., 2020) explain theoretically. Additionally, they find that countries with higher sovereign default risk experienced a higher decline in flows. Hence, we can interpret that the above mentioned uncertainty in capital markets is also reflected in their results.
Beside a macroeconomic perspective, a bunch of studies quest uncertainty during the crisis in terms of volatility and sentiment analysis.

For example, (Altig, et al., 2020) investigate economic (forward-looking) uncertainty indicators including implied volatility (1-month and 24-month VIX), both newspapers based political sentiment (Economic Policy Uncertainty Indices) and social sentiment about economic uncertainty (they construct a twitter-based economic uncertainty index), Business Expectation Panel Surveys (SBU for US and DMP for UK) and disagreement among one year GDP forecasts by professionals. The authors compare the measure between UK and US, before and during the crisis.

They summarize their results that most indicators reach their peaks during the pandemic (except 24-month VIX and GDP forecast disagreement). They also observe both a higher increase and sharper jumps in macro uncertainty compared to firm-level uncertainty. Moreover, they recognize that implied volatility increased sharply during late Februrary until mid of March and decreased within the following recovery period, whereas more macro-like indicators persist at a high level.

Another way is proposed by (Baker, et al., 2020), who compare the impact of historical pandemics on stock markets in a newspapers-based analysis and find that no other pandemic (for example SARS 2003 or Ebola 2015) had such a great effect on U.S. stock markets as COVID-19.

Alike (Altig, et al., 2020) they measure the role of news by automated and human readings of newspaper articles and conclude that the dominant driver of daily stock market jumps in the United States was due to news regarding the pandemic, interestingly in both directions (positive and negative moves). The authors filter jumps greater than $2.5 \%$ in absolute value and measure, if a next days' newspaper article makes the pandemic responsible for this jump. They observe 27 market jumps out of 41 trading days (February 24 to April 20) and report a jump frequency 20 times higher than average since 1900. They conclude that two dozen of jumps were related to the virus by newspaper.

(Baek, et al., 2020) research the impact of the crisis on U.S. stock market volatility by applying a Markov Regime-Switching AR model, combined with Machine Learning methods for feature selection across various industries. They find the largest shift in risk in the petroleum and natural gas industry, followed by restaurants, hotels and lodgings industry. Similar to (Alfaro, et al., 2020) they also mention that total risk of U.S. stock market is mostly influenced by positive and negative COVID-19 reports.

(Onali, 2020) investigates U.S. stock market behavior (Dow Jones, S\&P 500) during the pandemic using a $\operatorname{GARCH}(1,1)$, VARX and Markov Swiching models. His main result suggests that volatility is mainly affected by the change of cases and deaths, even they occurred abroad (Spain, Italy, Iran, China). However, there is no clear picture about the transmission channels.

(Zaremba, et al., 2020) examines the influence of non-pharmaceutical interventions during the crisis on stock market liquidity. They point out the importance of liquidity for the 
overall economy: Higher liquidity leads to lower cost of capital, contributes to a firm's financial resilience. They demonstrate that on the one hand workplace and school closures may limit stock market liquidity, but on the other hand public information campaigns fuel trading activity and so liquidity.

(Zaremba, et al., 2020) explain that the prime limitation of their study is the too short data set. This is due to the nature of the rather short period where markets struggled. They suggest using richer data sets like intraday data. (Zaremba, et al., 2020 )'s critique is among others exactly where we tie in with our analysis:

We are not interested in the influence of external factors like most authors are, but we are much more interested in the extent to which the American and European stock markets differ before, during and after the sharpe downward move in terms of uncertainty from the point of view of a pure time series analysis.

In times of crisis, market participants tend to react irrational and overhasty. Such a behavior leads to sharp spikes (jumps) and great uncertainty as shown by the studies above, what leads, in turn, to a even more leptokurtic distribution of returns. For this reason we need a model that is able to capture the observed properties and that's why we decided to use a jump diffusion model described in the next section.

Another point with which we contribute is that most studies we found deal with impact on U.S. stock markets only. To the best of our knowledge, this is the first work which takes both

Table 1. Descriptive Statistics for Euro Stoxx 50 and S\&P Index on intraday basis before, during and after the $\mathrm{V}$-shaped movement.

\begin{tabular}{|c|c|c|c|}
\hline & \multicolumn{3}{|c|}{ S\&P 500 } \\
\hline Period & $\mathbf{2 0 2 0 - 0 1 - 0 1 / 0 2 - 1 5}$ & $\mathbf{0 2 - 2 5 / 0 4 - 0 1}$ & $\mathbf{0 4 - 1 5 / 0 6 - 1 5}$ \\
\hline Length & 1266 & 1105 & 1730 \\
\hline Mean & $3.57026 \mathrm{e}-05$ & -0.00024 & $4.31391 \mathrm{e}-05$ \\
\hline Std & 0.00117 & 0.00781 & 0.00271 \\
\hline Skewness & -1.89919 & -2.26163 & 0.71670 \\
\hline Exc. Kurtosis & 61.95042 & 30.37088 & 31.14914 \\
\hline Min & -0.01792 & -0.08483 & -0.02673 \\
\hline Max & 0.01207 & 0.05551 & 0.02569 \\
\hline \multirow{2}{*}{ Euro Stoxx 50 } & \\
\hline Period & $\mathbf{2 0 2 0 - 0 1 - 0 1 / 0 2 - 1 5}$ & $\mathbf{0 2 - 2 5 / 0 4 - 0 1}$ & $\mathbf{0 4 - 1 5 / 0 6 - 1 5}$ \\
\hline Length & 1694 & 1431 & 2278 \\
\hline Mean & $1.49134 \mathrm{e}-05$ & -0.00022 & 3.17236 \\
\hline Std & 0.00102 & 0.00532 & 0.00240 \\
\hline Skewness & -0.84673 & -0.46624 & -0.89397 \\
\hline Exc. Kurtosis & 14.31771 & 8.998103 & 26.01811 \\
\hline Min & -0.00983 & -0.03958 & -0.02809 \\
\hline Max & 0.00676 & 0.03688 & 0.02186 \\
\hline
\end{tabular}

European and US Equity Markets into account as well as the examination of high frequency (10 minute) data.

For now, we continue with describing the methodology and data.

\section{DATA AND METHODOLOGY}

\subsection{Data}

For this investigation we use intradaydata of the Eurostoxx 50 Priceindex and S\&P 500 Index, where the first observable price each day is the official opening price and the last observable price is the official closing price. Hence, overnight movements are ignored in this analysis. The main motivation for calculating with intradaydata is the short time horizon the sharp downward move took place. Hence, we aggregated the data to $10 \mathrm{~min}$ data and returns are calculated as logarithmic returns. For a better computational tractability, we delete missing values as well as zeros from the return series.

For our purposes we divide the data set into three parts: before the crisis (2020-01-01/2020-02-15), during the crisis (202002-25/2020-04-01) and during the recovery period (2020-04$15 / 2020-06-15)$ ). We use disjoint time intervals so that there are no misleading overlappings in the model outcomes. We have chosen these subsamples by eye to cover the three mentioned periods.

Table 1 shows the descriptive statistics of the data (sub)set(s).

From the sample statistics we see that the mean is negative during the sharpe downturn and positive else. As expected, the standard deviation is highest during the downward period. This is due to higher uncertainty and panic reactions by market participants. But interestingly it remains on a higher level compared to before the down move.

The third and fourth sample moments indicate some interesting differences between US and European markets. The S\&P Index shows a lower negative skewness than the Euro Stoxx 50 Index during the first and second time window. Moreover, the level of skewness is way higher in US and even decreases during the downward shift, whereas in Europe we recognize an increasing skewness during this period. In the recovery period we see an even more drastic difference between the two markets. The American market turns positive in the recovery period (this means that we observe here a large frequency of occurrence of positve returns), whereas Europe turns even lower than before the downturn.

Also, excess kurtosis shows an always higher peakness in America compared to Europe. In combination, lower excess kurtosis and negative skewness shifts the distribution heavily to higher negative returns, which is plausible during the downtrend for both markets. The characteristic here is that the US market always has empirically higher excess kurtosis.

\subsection{The Model}

Not only the fact that equity returns are not normal distributed (see e.g. (Cont, 2001)), markets also show sharp spikes in terms of volatility and extreme returns during crises, as we have seen in descriptive statistics.

The above mentioned properties of asset returns lead to heavy tailed distributions. (Kou, 2002) proposed a model called 
Double Exponential Jump Diffusion (DEJD) Model, which accounts for such heavy tails.

Moreover, (Kou, 2002) outlines two properties regarding the Double Exponential Distribution, namely a highly skewed and leptokurtic distribution as well as the memoryless property, which is a realistic assumption for financial time series.

The model is described by

$d S(t)=S(t)\left(\mu d t+\sigma d W(t)+d \sum_{n=1}^{N(t)}\left(V_{n}-1\right)\right)$

where $\mathrm{S}(\mathrm{t})$ is the stock price at time $\mathrm{t}, \mu \mathrm{dt}$ is the drift term of the model and $\sigma$ is the standard deviation. $\mathrm{W}(\mathrm{t})$ describes a Wiener Process and $\mathrm{N}(\mathrm{t})$ is a Poisson Process with rate $\lambda$. The non negative i.i.d. sequence $V_{n}$ of random variables is chosen such that $Y=\log V_{n}$ has the density

$f_{Y}(y)=p_{u} \eta_{u} e^{-\eta_{u} y} \mathbf{1}_{y \geq 0}+p_{d} \eta_{d} e^{\eta_{d} y} \mathbf{1}_{y \leq 0}$.

Here, $\lambda$ from the Poisson Process is the total (positive and negative jumps) jump intensity, $p_{d}\left(p_{u}\right)$ serves as a measure for the probability that a jump is negative (positive) and it holds that $p_{d}+p_{u}=1 . \eta_{1}\left(\eta_{2}\right)$ is the characteristic lengths of a negative (positive) jump (see (Cont \& Tankov, 2009)).

The characteristic function of the DEJD model can be derived by applying the Lèvy-Khintchine formula. When a process exhibits finite variation jumps, the characteristic function of this process is given by

$\varphi(u)=\exp \left(t\left(i \mu u-\frac{1}{2} \sigma^{2} u^{2}+\int_{\mathbb{R}}\left(e^{i u y}-1\right) v(d y)\right)\right)$,

where $v$ is a Lèvy measure and gives the expected number of jumps in a unit time interval. In our case, the Lèvy density $v$ is given by $v=\lambda \phi(y)=\lambda\left(p_{u} \eta_{u} e^{-\eta_{u} y} \mathbf{1}_{y \geq 0}+\right.$ $p_{d} \eta_{d} e^{-\eta_{d} y} \mathbf{1}_{y \leq 0}$ ) (see (Mastro, 2013)).

\subsection{Estimation}

To estimate the model we use the Empirical Characteristic Function (ECF) Method. As described in (Yu, 2004), (Singleton, 2001) or (Rockinger \& Semenova, 2005) the idea of this method is basically to keep the distance between the model characteristic function (CF) and the empirical characteristic function as small as possible. In other words, we want to match the model CF and the empircal CF closely. To do so, we can formulate a target function which needs to be minimized

$\int_{-K}^{K}\left|\varphi_{\Theta}(u)-\hat{\varphi}(u)\right|^{2} g(u) d u$.

$\varphi_{\Theta}(u)$ is the characteristic function of the model containing the parameter vector $\Theta=\left(\mu, \sigma, \lambda, p, \eta_{u}, \eta_{d}\right)^{\prime}, \quad \hat{\varphi}(u)=$ $\frac{1}{T} \sum_{j=1}^{T} \exp \left(\mathrm{iu} X_{j}\right)$ is the empirical characteristic function and $g(u)$ is a (continuous) weighting function.

As described in (Tankov \& Voltchkova, 2009) the weighting function gives more weights to the tails of the distribution and is needed to ensure convergence and stability.
Following (Cont \& Tankov, 2009), we use

$(u)=\frac{\exp \left(-\widehat{\sigma}^{2} u^{2}\right)}{1-\exp \left(-\widehat{\sigma}^{2} u^{2}\right)}$

as a weighting function, where $\hat{\sigma}^{2}$ is the sample standard deviation.

Theoretically we calculate the integral in (4) over $\mathbb{R}$, but in practice, we truncate the integral and evaluate the integral on an interval $(-\mathrm{K}, \mathrm{K})$. This corresponds to removing very large jumps, but because the probability observing such large jumps is quite small it is an reasonable assumption. Also following (Cont \& Tankov, 2009), who did some simulation studies, where they figured out that the parameters are not sensitive to $\mathrm{K}>50$ anymore, we choose $\mathrm{K}=60$.

\section{RESULTS AND INTERPRETATION}

Table $\mathbf{2}$ and $\mathbf{3}$ show the results of the parameter estimation.

When looking at the estimated mean value of the model $\mu$ (drift) we see that there is no good fit with respect to the sample mean. The model estimates a negative mean after the crisis for both markets. Moreover, the drift value for the European stock market is estimated to be positive during the crisis.

However, because of the complexity of the model and values close to and around zero it is difficult to estimate the drift term accurately ${ }^{1}$.

That's why we have a closer look at the remaining variables.

In both Europe and USA we observe a sharp increase of volatility during the crisis compared to the period before and a decrease afterwards but a higher level compared to the first period of interest.

This result seems plausible, especially the higher level of volatility during the downward move. This is a typical pattern during crises. We also see that the volatility is approximately equal before, but higher in the US market during the crisis and equal again afterwards.

A similar picture gives the jump intensity $\lambda$.We detect that the jump intensity increased significantly during the period of crisis also in both regions. This coincides with the hypothesis that during crises the level of uncertainty rises and market participants tend to overreact. However, we obvserve a difference between Europe and USA in the sense that the intensity remains on a higher level in the US after the downspike. Looking at the intraday prices in figure 2 we see that the upward move (last panel/row) is more noisy in the US than in Europe, what can be interpreted as a confirmation of the estimate. This result basically matches the results found in (Onali, 2020) and (Baker, et al., 2020), since one can connect high volatility driven by more and more intense jumps with fear of the virus.

According to the model we recognize a downward jump probability (p) of $65 \%, 53 \%, 38 \%$ in Europe and $37 \%, 31 \%, 42$ $\%$ in the US. 

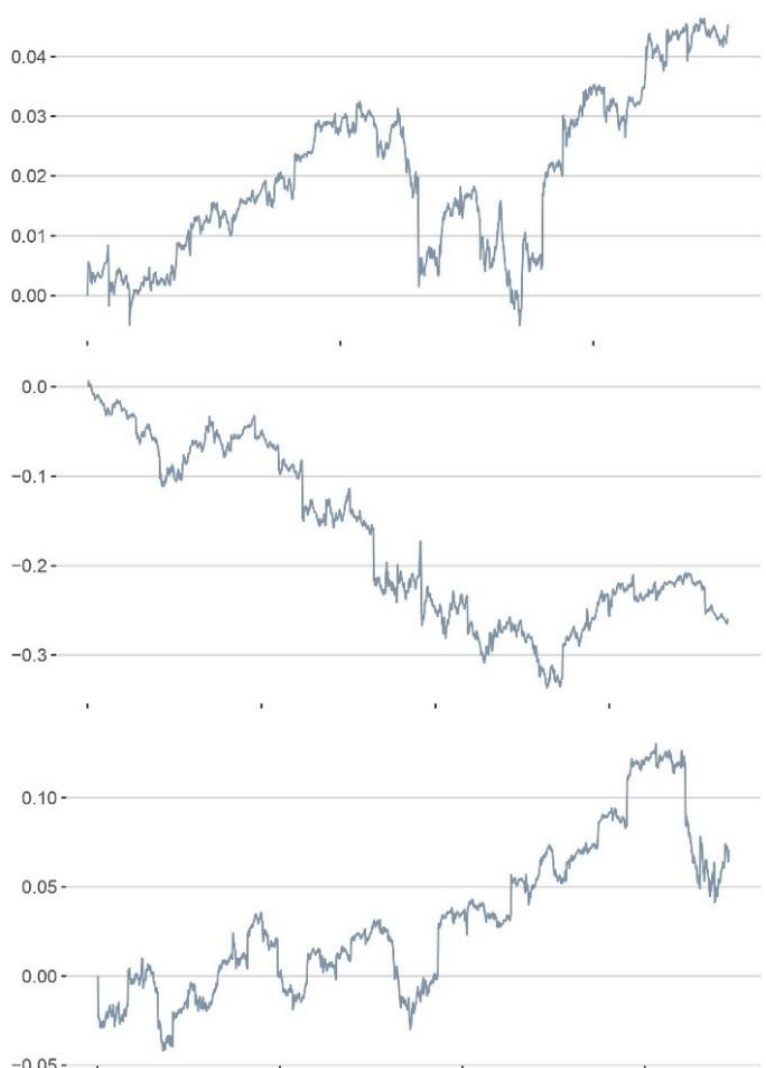
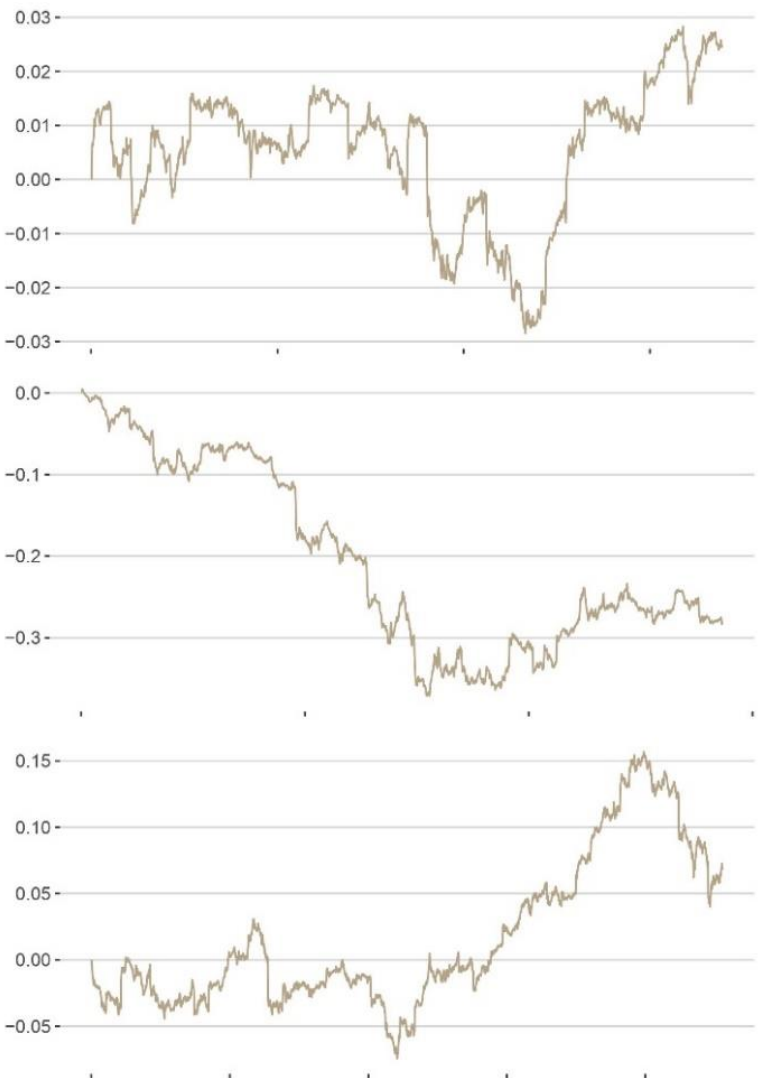

Fig. (2). Scaled intraday prices of S\&P Index (left) and Eurostoxx 50 (right) for time periods per row from first to last 2020-01-01/2020-02$15,2020-02-25 / 2020-04-01,2020-04-15 / 2020-06-15$.

In a first glance this result seems counterintuitive, but in the case of Europe we see in Fig. (2) (right hand side) above that there was great turmoil before the outbreak of the pandemic, which makes it plausible that the probability of a downward jump is large. This probability decreases during the pandemic crisis and is reduced again after the crisis. This means, we observe a downtrend during the crisis, but this downtrend is not followed by many downward jumps, but is rather smooth. The same statement is applicable in reverse for the upward period, what can be seen in Fig. (2).

Compared to the existing literature one can connect this result with the analysis of (Baker, et al., 2020), who reports jumps in both directions.

Similar, the probability of a downward jump falls when we entering the crisis state with about the same magnitude as Europe. For the USA we face a lower downjump probability during the crisis but a higher one in the recovery. Comparing this observation with the chart above, the estimate becomes also a plausible result.

We observe here a big difference between the two markets. It seems that market participants in Europe have been more cautious (especially when additional considering jump intensity) than American investors. With this we find one reason, why the S\&P Index did better in the recovery period compared to the SX5E Index, what can be clearly seen in Fig. (1).

$\eta_{i}, i=u, d$ gives the characteristic length of a positive $(\mathrm{i}=\mathrm{u})$ or negative $(i=d)$ jump. We see that in all cases it holds that $\eta_{d}<\eta_{u}$. This means that a negative jump has a shorter duration compared to a positive one. Also remarkable, in both markets and both directions (positive and negative jumps) the characteristic length increases during the crisis and remains on a higher level after the crisis. This finding also coincide with those in (Baker, et al., 2020). Interestingely, the characteristic length of a downward jump is shorter than the length of an upward jump in most cases. This agrees with the common wisdom that the stock market goes up like staircase but down like an elevator.

Table 2. Parameter Estimates from calibrating the (Kou, 2002) Model for SX5E Index.

\begin{tabular}{|c|c|c|c|}
\hline Parameter & $\begin{array}{c}\text { Before crisis } \\
(2020-01- \\
01 / 2020-02-15)\end{array}$ & $\begin{array}{c}\text { During crisis } \\
(2020-02- \\
25 / 2020-04-01)\end{array}$ & $\begin{array}{c}\text { After crisis } \\
(2020-04- \\
15 / 2020-06-15)\end{array}$ \\
\hline$\mu$ & 0.00053 & 0.00029 & $-4.87273 e-05$ \\
\hline$\sigma$ & 0.00180 & 0.00631 & 0.00293 \\
\hline$\lambda$ & 0.91958 & 3.92993 & 0.69366 \\
\hline$p$ & 0.65830 & 0.53036 & 0.38129 \\
\hline$\eta_{u}$ & 0.00151 & 0.00525 & 0.00475 \\
\hline$\eta_{d}$ & 0.00146 & 0.00479 & 0.00343 \\
\hline
\end{tabular}


Table 3. Parameter Estimates from calibrating the (Kou, 2002) Model for SPX Index.

\begin{tabular}{|c|c|c|c|}
\hline Parameter & $\begin{array}{c}\text { Before crisis } \\
\mathbf{( 2 0 2 0 - 0 1 -} \\
\mathbf{0 1 / 2 0 2 0 - 0 2 - 1 5 )}\end{array}$ & $\begin{array}{c}\text { During crisis } \\
\mathbf{( 2 0 2 0 - 0 2 -} \\
\mathbf{2 5 / 2 0 2 0 - 0 4 - 0 1 )}\end{array}$ & $\begin{array}{c}\text { After crisis } \\
\mathbf{( 2 0 2 0 - 0 4 -} \\
\mathbf{1 5} / \mathbf{2 0 2 0 - 0 6 - 1 5})\end{array}$ \\
\hline$\mu$ & 0.00024 & -0.00563 & -0.00060 \\
\hline$\sigma$ & 0.00180 & 0.00810 & 0.0022 \\
\hline$\lambda$ & 0.60498 & 2.76158 & 1.31905 \\
\hline$p$ & 0.37260 & 0.30579 & 0.42044 \\
\hline$\eta_{u}$ & 0.00353 & 0.01328 & 0.00442 \\
\hline$\eta_{d}$ & 0.00237 & 0.00784 & 0.00439 \\
\hline
\end{tabular}

To put it in a nutshell, although we observe a similar behavior of the time series with the naked eye, the underlying structure seems to be quite different between US and Europe. The two markets coincide in terms of the path of volatility, jump intensity as well as characteristic lengths of positive and negative jumps, but do not coincide in terms of jump probabilities.

Nevertheless, as (Altig, et al., 2020) we also find higher uncertainty in the recovery period also for Europe as well as a higher jump intensity during the sharpe downturn.

Hence, we contribute to the literature such that we show similar results as existing studies, but from a pure time series approach.

\section{CONCLUSION}

This paper investigates and compares the behavior of European and American stock markets applying (Kou, 2002)'s Double Exponential Model to intraday data before, during and after the worldwide Corona pandemic. Using this model we can compute jump intensity, probabilities of up and downward jumps as well as characteristic lengths of those jumps. Hence, we are able to compare and analyze similarities and differences between the two markets in the above mentioned time period.

Allthough the market movements seem quite similar, the main finding is that the two stock markets are distinct from each other regarding the probabilities of downward jumps.

Downward jumps were more probable in Europe than in USA during the crisis. Nevertheless, the intensity of jumps rose in both markets significantly during the drawdown and stayed on a higher level compared to before the crisis. However, characterisitc lengths of jumps behaved quite similar.

This is a very interesting result, because this means that the overall uncertainty was higher in Europe than in the US. We can carefully conclude that one reason why the US market recovered better lies in this difference of uncertainty. A possible implication for Asset Manager managing a trading Portfolio containing both European and US markets is that stop losses should have been wider for the Eurostoxx 50. Additionally, for Asset Manager following allocation strategies should have included an adjustment factor to consider the higher probability of (downward) jumps in Europe.
All in all we contribute to the literature by being the first who apply a pure time series model to European and American stock market data before, during and after the corona crisis. Nevertheless we get similar results (especially in terms of jump analysis) as (Altig, et al., 2020) from this time series perspective.

However, further research is needed for an analysis on a longer time horizon after the stark drawdown to investigate if jump probability and intensity changed or normalized to a pre crisis level. Another point is to compare the behavior of markets during different crisis to check if there is a systematic difference in both markets.

\section{A. TECHNICAL REMARKS}

For preparing high frequency data we use the R package high frequency.

We tried several optimization routines from PORT library with different optimization algorithms. In R, the function nlminb worked best. Using nlminb we got the lowest function value compared to optim (L-BFGS-B), nloptr in the nloptr package (Newuoa) or fminsearch in the pracma package (Hooke-Jeeves). We also checked parameter robustness by trying different initial values.

It seems that the model is very sensitve to initial values. For example we didn't find a global minimum when we started with a too low $\lambda$ in the downward period. We have chosen the initial values with an educated guess as follows: $\mu=0.001, \sigma$ $=0.001, \lambda=0.5, \mathrm{p}=0.5, \eta_{u}=0.05, \eta_{d}=0.05$.

\section{CONFLICT OF INTEREST STATEMENT}

The authors declare that they have no conflict of interest.

\section{REFERENCES}

Alfaro, Laura, Anusha Chari, Andrew Greenland, und Peter Schott. „Aggregate and Firm-Level Stock Returns during Pandemics, in Real Time." National Bureau of Economic Research, 2020.

Altig, Dave, et al. „Economic Uncertainty before and during the COVID-19 Pandemic." Journal of Public Economics, 191, 2020.

Baek, Seungho, Sunil Mohanty, und Mina Glambosky. „COVID-19 and Stock Market Volatility: An Industry Level Analysis." Finance Research Letters, 2020.

Baker, Scott R, Nicholas Bloom, Steven Davis, Kyle Kost, Marco Sammon, und Tasaneeya Viratyosin. „The Unprecedented Stock Market Reaction to COVID-19." The Review of Asset Pricing Studies, 10(4), 2020: 742-758.

Cont, Rama. „Empirical Properties of Asset Returns: Stylized Facts and Statistical Issues.“ Ouantitative Finance, 1, 2001: 223-236.

Cont, Rama, und Peter Tankov. „Constant Proportion Portfolio Insurance in the Presence of Jumps in Asset Prices." Mathematical Finance, 19(3), 2009: 379 - 401

Czeczeli, Vivien, Peter Kolozsi, Gabor Kutasi, und Adam Marton. „Economic Exposure and Crisis Resilience in Exogenous Shock: The Short-Term Economic Impact of the Covid-19 Pandemic in the EU." PÉNZÜGYI SZEMLE/PUBLIC FINANCE QUARTERLY, 65(3), 2020: 321-347.

ElFayoumi, Khalid, und Martina Hengge. „Capital Markets, COVID-19 and Policy Measures.“ International Monetary Fund, 2021.

Kou, Steven. „A Jump-Diffusion Model for Option Pricing.“ Management Science, 48(8), 2002: 1086-1101.

Mastro, Michael. Financial Derivative and Energy Market Valuation. Wiley Online Library, 2013.

Onali, Enrico. „Covid-19 and Stock Market Volatility.“ Available at SSRN 3571453, 2020.

Rockinger, Michael, und Maria Semenova. „Estimation of Jump-Diffusion Processes via Empirical Characteristic Functions." Available at SSRN 770785, 2005 
Sheiner, Louise, und Kadija Yilla. ,The ABCs of the Post-COVID Economic Recovery." BROOKINGS BRIEF, 2020.

Singleton, Kenneth. „Estimation of Affine Asset Pricing Models Using the Empirical Characteristic Function." Journal of Econometrics, 102(1), 2001: 111-141.

Tankov, Peter, und Ekaterina Voltchkova. „Jump-diffusion Models: A Practitioner's Guide.“ Banque et Marchés, 99(1), 2009.

World Bank. „Global Economic Prospects, January 2021.“2021.
Yu, Jun. „Empirical Characteristic Function Estimation and its Applications.“ Econometric Reviews, 23(2), 2004: 93-123.

Zaremba, Adam, David Aharon, Ender Demir, Renatas Kizys, und Dariusz Zawadka. „COVID-19, Government Policy Responses, and Stock Market Liquidity around the World: A Note." Research in International Business and Finance, 2020.

Received: May 02, 2021

Copyright (C 2021- All Rights Reserved

This is an open-access article. 